\title{
Allelic mutations of the sodium channel SCN8A reveal multiple cellular and physiological functions
}

\author{
Miriam H. Meisler, Nicholas W. Plummer, Daniel L. Burgess, David A. Buchner \& \\ Leslie K. Sprunger \\ Department of Human Genetics, University of Michigan, Ann Arbor, MI 48109-0618, USA (Phone: + 1-734- \\ 763-5546; Fax: + 1-734-763-9691; E-mail: meislerm@umich.edu); Current addresses: N.W. Plummer, \\ NIEHS, Research Triangle Park NC; D.L. Burgess, Department of Neurology, Baylor College of Medicine, \\ Houston TX; D.A. Buchner, Life Sciences Institute, University of Michigan, Ann Arbor MI; L.K. Sprunger, \\ College of Veterinary Medicine, Washington State University, Pullman, WA
}

Key words: allelic series, hypomorphs, med, missense mutations

\begin{abstract}
Allelic mutations of Scn8a in the mouse have revealed the range of neurological disorders that can result from alternations of one neuronal sodium channel. Null mutations produce the most severe phenotype, with motor neuron failure leading to paralysis and juvenile lethality. Two less severe mutations cause ataxia, tremor, muscle weakness, and dystonia. The electrophysiological effects have been studied at the cellular level by recording from neurons from the mutant mice. The data demonstrate that Scn8a is required for the complex spiking of cerebellar Purkinje cells and for persistent sodium current in several classes of neurons, including some with pacemaker roles. The mouse mutations of Scn8a have also provided insight into the mode of inheritance of channelopathies, and led to the identification of a modifier gene that affects transcript splicing. These mutations demonstrate the value of mouse models to elucidate the pathophysiology of human disease.
\end{abstract}

\section{Overview}

The spontaneous mouse neurological mutant 'motor endplate disease' (med) arose in 1958 at the University of Edinburgh (Duchen, Searle \& Strich, 1967). This autosomal recessive mutation resulted in muscle atrophy, paralysis and death. Two allelic mutations were later identified at the Jackson Laboratory, med ${ }^{J}$ and jolting (Sidman, Cowen \& Eicher, 1979). Elegant morpholgical, physiological and electrophysiological studies in the laboratories of Duchen and Harris in the 1970s and 1980s led to the prediction that the mutation affected an ion channel gene involved in release of neurotransmitter from the nerve terminals of motor neurons. In 1992, an insertional mutation with a similar phenotype was discovered by Brett Spear at the University of Kentucky. We demonstrated allelism of the new mutation by noncomplementation with med $^{J}$, and demonstrated that the disrupted gene was a novel neuronal sodium channel (Burgess et al., 1995; Kohrman et al., 1995). Fortunately, the first three alleles had been preserved, and Dave Kohrman went on to identify the molecular lesions in all three alleles (Kohrman, Harris \& Meisler, 1996a; Kohrman et al., 1996b). The Scn8a allele series provides the first demonstration of the in vivo physiological and clinical consequences of mutations in a neuronal sodium channel gene. In addition to the visible neurological disorders, electrophysiological studies of neurons isolated from these mice have revealed some of the unique cellular functions of the Scn8a channel. The phenotypes of the mouse mutants provide a prediction of the types of human patients that may carry mutations in voltage gated sodium channels. The first such mutation was found in our laboratory earlier this year (Trudeau et al., 2000). The Scn8a 
allele series demonstrates the heterogeneity of disorders resulting from different mutations in a single gene, and illustrate the value of multiple alleles for elucidating cellular and physiological function.

\section{The voltage-gated sodium channel $\alpha$ subunit gene family}

The voltage-gated sodium channels are responsible for the rising phase of the action potential in neurons and muscle cells. The mammalian genome contains 10 closely related sodium channel genes with highly conserved protein sequence but divergent tissue-specific expression. These genes are located in four paralogous chromosome segments that also contain the HOX gene clusters (Plummer $\&$ Meisler, 1999). Four of the sodium channel genes are expressed at a high level in the central nervous system (CNS): $S C N 1 A, S C N 2 A, S C N 3 A$, and $S C N 8 A$. Most neurons in the CNS appear to express all of these channels, albeit in different proportions. Although their in vitro channel biochemical activities are very similar, null alleles of Scn1a, Scn2a and Scn8a in the mouse are all lethal, demonstrating that they are not functionally redundant in vivo (Burgess et al., 1995; PlanellsCases et al., 2000; W. Catterall, pers. commun.). Analysis of rescue by chimeric channels may provide insight into the unique functions associated with each family member. Physiological studies of these mice will be essential for understanding the role of each channel in the intact nervous system.

\section{Characteristics of sodium channel SCN8A}

SCN8A is one of the most abundant voltage-gated sodium channels in the brain (Schaller et al., 1995), and is the major channel at the nodes of Ranvier in mature myelinated axons (Caldwell et al., 2000; Krzemien et al., 2000; Schaller \& Caldwell, 2000; Tzoumaka et al., 2000). Additional sites of subcellular localization in the CNS include dendrites, presynaptic and postsynaptic membranes, and nonmyelinated axons. Because it is found at synapses, modulation of SCN8A activity by genetic variation or physiological modification may be expected to influence synaptic strength, learning and memory.

\section{An insertional allele of the $S c n 8 a$ locus, $S c n 8 a^{t g}$}

The insertional mutation of $S c n 8 a$ arose during a long-term study of expression of the alpha fetoprotein promoter in transgenic mice (Spear, 1994; Ramesh, Ellis \& Spear, 1995). Homozygous transgenic mice of one line, A4, exhibited a neurological syndrome that began with abnormal gait at 2 weeks of age and led to paralysis and death by 3 weeks of age. The new mutation was genetically mapped approximately $10 \mathrm{cM}$ distal to the position of the med locus on the consensus map of mouse chromosome 15 (Kohrman et al., 1995). Because of the similarity in the phenotypes of A4 and med, we tested for alleleism by crossing A4 transgenic heterozygotes with heterozygous med $^{J}$ mice from the frozen embryo bank at The Jackson Laboratory. The expected frequency of $25 \%$ of compound heterozygote offspring were recovered in the F2, and these animals displayed the typical med phenotype, demonstrating that the new mutation was allelic with med $^{J}$.

\section{Cloning of the Scn8a gene}

To identify the mutated gene, we generated a cosmid library of genomic DNA from Scn $8 a^{t g}$ homozygotes and screened it by hybridization with the transgene; the methods were described (Meisler et al., 1997) and are still appropriate for the isolation of new insertional mutants. Junction clones containing both transgene and adjacent DNA from the insertion site were analyzed and found to contain exons from a novel sodium channel (Burgess et al., 1995). A deletion of $\leq 20 \mathrm{~kb}$ in the middle of the Scn8a gene removed several exons and inactivated the gene.

The human ortholog of the new gene, SCN8A, was isolated and mapped to a conserved linkage group on chromosome 12q13 (Burgess et al., 1995). The structure and sequence of the human gene have been determined (Plummer et al., 1998). The orthologous rat cDNA was independently isolated in 1995 (Schaller et al., 1995), and the human cDNA was subsequently isolated from dorsal root ganglion neurons (Dietrich et al., 1998). This channel has been variously designated NaCh6, CerIII and PN4; the new recommended nomenclature is $\mathrm{Na}_{\mathrm{v}} 1.6$. 
The original med mutation is a null allele of $S c n 8 a$

The original med line and the jolting allele were maintained by Dr John Harris at the Neuromuscular Disease Unit in Newcastle, UK, for more than 20 years, and he kindly provided breeding pairs. Analysis by RT-PCR and genomic sequencing demonstrated that the original med mutation was caused by insertion of a transposable $\mathrm{L} 1$ element into exon 2 (Figure 1). Aberrant splicing of the mutant transcript disrupts the open reading frame and truncates the protein close to the $\mathrm{N}$-terminus (Kohrman, Harris \& Meisler, 1996a). Thus both the original allele and the transgene insertion allele are nulls, and exhibit identical phenotypes (Table 1).

Failure of motor nerve function in Scn8a null mice results in juvenile lethality

Homozygous $S c n 8 a^{\text {med }}$ and $S c n 8 a^{\text {medtg }}$ mice develop severe muscle atrophy, weakness, and progressive paralysis that begins in the hind limbs. Between 17 and 23 days of age, the sciatic nerve exhibits reduced conduction velocity, prolonged refractory period, and widening of the non-myelinated gaps at the nodes of Ranvier (Agnaut-Petit et al., 1982; Rieger et al., 1984; Bournaud et al., 1987; Füchtbauer, 1987; Kearney, 2002). These changes are accompanied by failure of transmission at the neuromuscular junction, with somewhat faster progression in biceps than in triceps (Duchen \& Stefani, 1971). Terminal sprouting of the motor nerves, muscle fiber atrophy, and appearance of central nuclei indicative of fiber regeneration (Duchen, Searle \& Strich, 1967; Duchen \& Stefani, 1971) demonstrate that the motor neurons do not properly innervate the muscle in homozygous null mice, which do not survive beyond 1 month of age.

The severe phenotype and hind limb paralysis of the null mutants can be understood in the light of recent evidence that $\mathrm{Scn} 8 \mathrm{a}$ is the major channel at the nodes of Ranvier (Caldwell et al., 2000). The total sodium current of motor neurons increases three fold during the first week of life in normal mice, and most of this increase appears to be due to Scn8a, since there was no increase in Scn8a null mice (Garcia et al., 1998). Scn8a is also the major nodal channel in nerves innervating the diaphragm muscle, and paralysis of the diaphragm may be the cause of death. Because of the early lethality, the null mutant cannot be used to study the effects of Scn8a deficiency in adult brain. Heterozygotes for the null mutations are phenotypically normal, demonstrating that $50 \%$ of normal levels of Scn8a is adequate.

\section{A hypomorphic allele of $S c n 8 a$ results in severe muscle weakness and dystonic postures}

The med $^{J}$ mutation arose 30 years ago at the Jackson Laboratory in a linkage stock carrying the closely linked visible marker Caracul $(\mathrm{Ca})$. A 4 bp deletion in the splice donor site of intron 3 results in abnormal splicing and skipping of exons 2 and 3 (Kohrman, Harris \& Meisler,

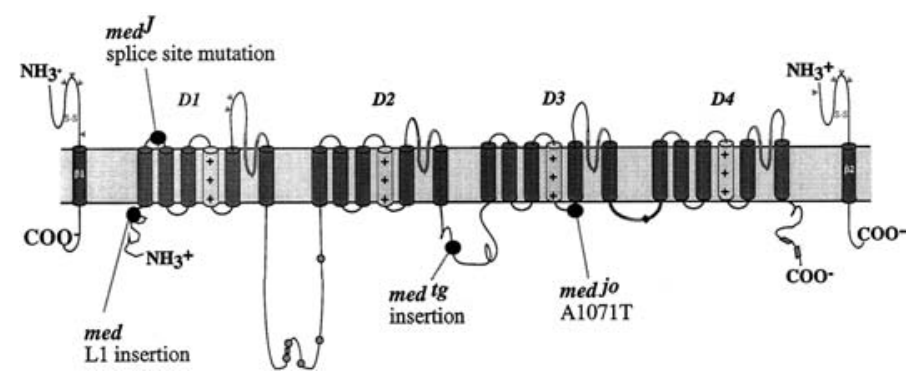

Figure 1. Positions of four allelic mutations of the mouse sodium channel Scn8a. The voltage-gated sodium channels contain four homologous domains (D1 to D4) each with six transmembrane segments (S1 to S6). The domains are separated by cytoplasmic loops and a short inactivation linker. The sequences of the transmembrane domains are more conserved than the cytoplasmic loops. The positively charged S4 segments act as voltage sensors and move outward from the membrane in response to depolarization. The associated transmembrane $\beta 1$ and $\beta 2$ subunits are also shown. 
1996a). The resulting protein is truncated in Domain 1 (Figure 1). However, Scn $8 a^{\text {medJ } J}$ is not a true null allele because approximately $12 \%$ of the transcripts are correctly spliced (Kearney et al., 2002). This low level of expression prevents paralysis, but homozygotes have severe muscle weakness and cannot support their own weight. They also exhibit frequent episodes of sustained abnormal postures of the limbs and body (Sprunger et al., 1999). These dystonic postures persist for periods from 5 to $10 \mathrm{~s}$ in young animals, and for several minutes in older animals. An example is shown in Figure 2, left panel. These postures resemble human dystonia, although it is not known whether the pathogenesis is of central or peripheral origin. Imbalance in the strength of flexor and extensor muscles could be responsible, but abnormal currents in higher centers of motor control may also contribute the dystonic phenotype. A 'floxed' allele of Scn8a has been developed, in order to resolve these alternatives using tissue specific inactivation of the channel (S. Levin \& M. Meisler, genesis 2004). Inactivation of $S c n 8 a$ in cerebellar Purkinje cells reproduces a subset of the null phenotype (manuscript in preparation).

\section{A missense mutation of $S c n 8 a$ results in tremor and ataxia}

The jolting mutation arose at the Jackson Laboratory in 1965 (Sidman, Cowen \& Eicher, 1979). A single nucleotide change results in an alanine to threonine substitution in an evolutionarily conserved cytoplasmic linker domain (Figure 1) (Kohrman et al., 1996b). This mutation causes a $14 \mathrm{mV}$ positive shift in the voltage dependence of activation of the channel (Kohrman et al., 1996b; Smith \& Goldin, 1999). Scn $8 a^{j o}$ homozygotes have chronic ataxia with an unsteady, wide-based gait, as well as a rhythmical tremor of head and neck that is induced by attempted movement (Dick, Boakes \& Harris, 1985). Neuromuscular transmission is normal, and the mice are fertile and have a normal lifespan. Cerebellar Purkinje cells show a lack of spontaneous activity and there is progressive loss of Purkinje cells after 6 months of age (Dick, Boakes \& Harris, 1985; Harris, Boakes \& Court, 1992; see below). The characteristics of the four Scn8a mutants are summarized in Table 1. 


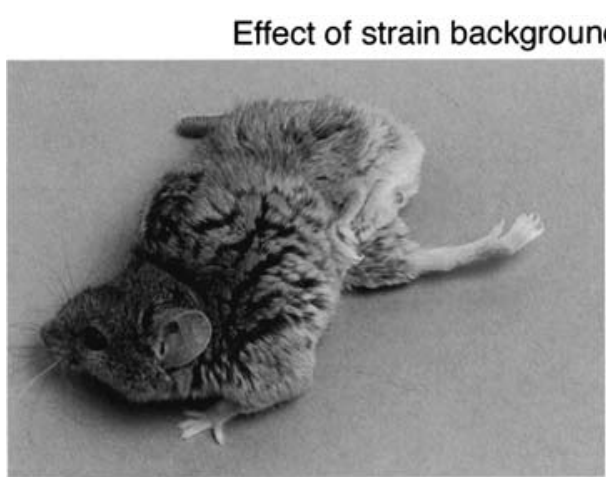

$\mathrm{C} 3 \mathrm{H}$

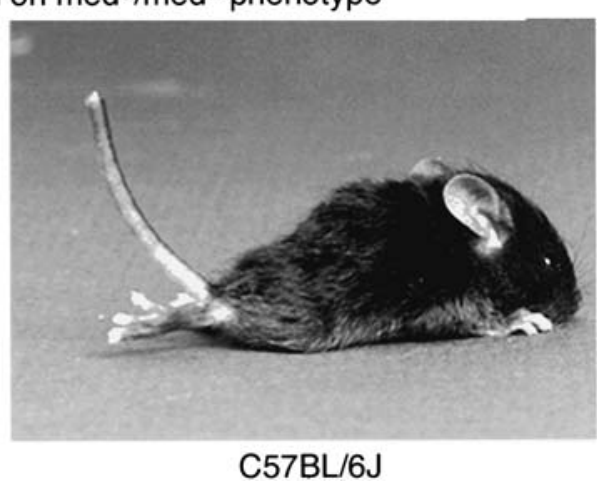

C57BL/6J

Figure 2. A modifier gene alters the clinical consequences of low levels of Scn8a in homozygous med ${ }^{J}$ mice. The 8 month old homozyogte on strain $\mathrm{C} 3 \mathrm{H}$ exhibits muscle weakness and dystonic postures. The 3 weeks old animal on strain $\mathrm{C} 57 \mathrm{BL} / 6 \mathrm{~J}$ is paralyzed. The difference is caused by the Scnm1 modifier locus on chromosome 3.

\section{Scn8a is essential for complex spiking in cerebellar Purkinje cells}

The most striking cellular effect of the Scn8a mutations described to date is the effect on cerebellar Purkinje cells. These cells are distinguished by their spontaneous slow firing pattern and their ability to generate a series of action potentials after stimulation. This complex spiking activity is thought to be involved in integration of signals for motor control. Recordings of currents from Purkinje cells demonstrate loss of complex spiking in both $S c n 8 a^{t g}$ and $S c n 8 a^{j o}$ mice (Figure 3, right panel). The similar effect of the null and the missense mutation indicates that the Purkinje cell is particularly dependent on Scn8a. This functional requirement is consistent with the high level of Scn8a expression that was detected in Purkinje cells (Schaller et al., 1995; Krzemien et al., 2000). Scn8a mutations also affect two other characteristic
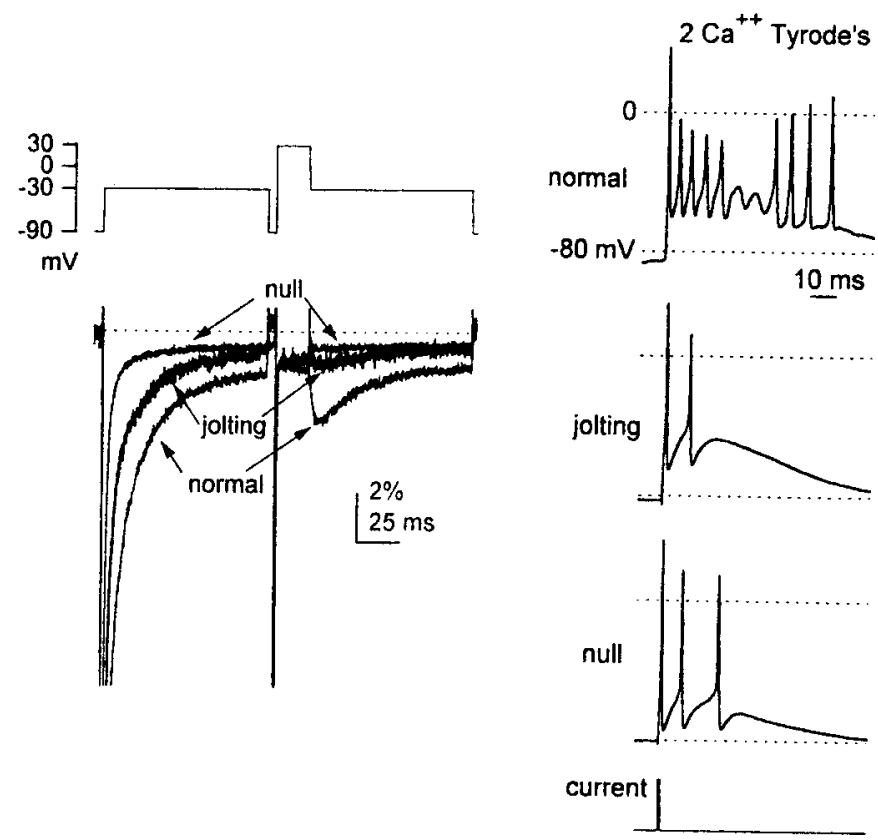

Figure 3. Two characteristic currents of cerebellar Purkinje cells are reduced in mice with mutation in Scn8a. Left, resurgent current and persistent currents; right, complex spiking. Reprinted from Raman et al. (1997) with permission of the publisher. 
sodium currents of Purkinje cells, the persistent current and the resurgent current (Figure 3, left panel) (Raman et al., 1997). It is interesting that the small change in voltage dependence of $S c n 8 a^{j o}$ has a profound effect on currents in Purkinje cells but does not impair the generation of action potentials in motor neurons.

Cartwheel cells of the dorsal cochlear nucleus are ontologically related to cerebellar Purkinje cells and exibit similar firing patterns. Currents recorded from the dorsal cochlear nucleus in brain slices from $S c n 8 a^{\text {medJ }}$ and $S c n 8 a^{j o}$ mice demonstrated extensive loss of these cells as well (Chen et al., 1999). Persistent current is a feature of many pacemaker neurons and pyramidal cortical cells, and the med mutants alter these as well (Raman et al., 1997; Maurice et al., 2001).

\section{Threshold quantitative requirement for SCN8A: how much is enough?}

For most enzyme deficiencies, a few percent of normal activity is sufficient to prevent disease, but for structural proteins like collagen, haploinsufficiency is common. We generated mice with various levels of Scn8a by combining different alleles (Table 2). The results suggest that the critical threshold for survival is between 6 and $12 \%$ of normal levels, and the threshold for normal movement is between 12 and $50 \%$ of the normal concentration.

\section{Dominant or recessive inheritance of sodium channel mutations}

The mode of inheritance of ion channel mutations is determined by the nature of the channel defect

Table 2. Quantitative threshold for SCN8A function (+, wildtype; -, null)

\begin{tabular}{lll}
\hline Genotype & Amount & Phenotype \\
\hline$+/+$ & $100 \%$ & Normal \\
$+/-$ & $50 \%$ & Normal \\
med $^{J} /$ med $^{J}$ & $12 \%$ & Dystonia, muscle \\
& & weakness \\
med $^{J} /-$ & $6 \%$ & Lethal \\
$-/-$ & 0 & Lethal \\
\hline
\end{tabular}

and the function of the mixed channel population in heterozygous cells. Sodium channel $\alpha$ subunits function as monomers. Many human mutations in ion channels result in incomplete inactivation of the channel and are inherited as dominant disorders (Bulman, 1997). In contrast, the $S c n 8 a$ alleles in the mouse are recessive. This can be understood in terms of the functional effects of each allele. $S c n 8 a^{j o}$ encoded channels require an extra $14 \mathrm{mV}$ of depolarization before the channel is activated. In heterozygotes, the wildtype channels activate at normal voltage and can initiate the action potential. Thus both types of inheritance may occur, depending on the functional characteristics of the mutant protein.

\section{Digenic inheritance: an unlinked modifier locus of the hypomorphic $S c n 8 a^{\text {medJ }}$ allele}

The influence of secondary genetic factors on the severity of human monogenic disorders is becoming increasingly evident (Dipple \& McCabe, 2000). Inbred strains of mice carry different alleles at many loci. Interacting genes that modify a mutant phenotype may be identified by crossing a mutant onto different strains. The $S c n 8 a^{\text {medJ }}$ mutation has been tested on five strain backgrounds. Muscle weakness and dystonic postures were observed on four strains: C3H, DBA, A/J and 129/SvJ (Figure 2, left panel). However, on strain C57BL/6J, $S c n 8 a^{\text {medJ }}$ homozygotes mimic null homozygotes and do not survive (Figure 2, right panel). A modifier gene on chromosome 3 , designated sodium channel modifier 1 (Scnml), co-segregates with lethality in crosses between C57BL/6J and each of the other strains (Sprunger et al., 1999, Buchner et al., 2003a). Thus C57BL/6J appears to carry a unique, 'susceptible' allele of the modifier Scnm1. The orthologous human chromosome band 1q21 is a gene-dense region containing several genes with neuronal functions. We used positional cloning to identify this novel gene and its mechanism of interaction with Scn8a (Buchner, Trudeau \& Meisler, 2003b). The modifier gene encodes an U1C zinc finger protein with nuclear localization that acts on the ed $^{J}$ transcript to increase the proportion of correctly spliced transcripts. The isolation of $S C N M 1$ is one of the first demonstrations of the mechanism of interaction of a modifier gene with a primary disease gene. 
Insights from multiple alleles of $S c n 8 a$

If we had been limited to analysis of a null allele of Scn $8 a$, we would not have guessed that mutations in this gene could result in tremor, ataxia, or dystonia, all serious human medical concerns. We would also have been unable to study the role of the channel in the adult nervous system. Within the next few years we anticipate a significant increase in the number of human neurological disorders associated with sodium channel mutations. We are likely to discover a heterogeneous group of amino acid substitutions causing mild alterations in channel properties, like the SCN1A mutations in GEFS plus (Escayg et al., 2000). Because of the critical role of sodium channels in neuronal signaling, structural variants may also be susceptibility factors in polygenic disease, including the common psychiatric disorders, through genetic interaction with subtle variants in other proteins. We have identified rare coding variants of SCN1A, SCN2A and SCN3A in 5\% of patients with familial autism (Weiss et al., 2003). Different classes of neurons may vary in their susceptibility to specific mutations, as we have seen for the jolting mutation in Purkinje versus motor neurons.

The phenotypes of $S c n 8 a$ mutant mice have directed our attention to specific human patients for mutation testing. We anticipate that at some point in the future all admissions to neurological or psychiatric services will be routinely screened for channelopathies, using a sequencing chip that can detect variation in all 10 sodium channel genes. A wealth of information on structure/ function relationships will then become available. Introduction of human disease mutations into the orthologous mouse gene as transgenes or targeted mutation will be useful for verifying causality and for analysis of pathogenic mechanisms (Figure 4). Recordings from neurons isolated from mutant mice provide data about mutant channels that goes beyond the information that can be obtained from expression in Xenopus oocytes or transfected mammalian cells. Crosses with mice carrying mutations in other genes can reveal genetic interactions and modifiers influencing clinical phenotypes. Analysis of multiple alleles in the mouse can provide a depth of understanding of in vivo gene function that is otherwise unavailable.

\section{Acknowledgements}

Our work on the alleles of $S c n 8 a$ has been supported by research grants from the National Institute of General Medicine (GM24872), the National Institute of Neurological Disease and Stroke (NS34509), the March of Dimes and the Wilson Medical Research Foundation.

\section{References}

Angaut-Petit, D., J.J. McArdle, A. Mallart, R. Bournaud, M. Pincon-Raymond \& F. Riegers, 1982. Electrophysiological and morphological studies of a motor nerve in 'motor endplate disease' of the mouse. Proc. Royal. Soc. Lond. B Biol. Sci. 215: 117-125.

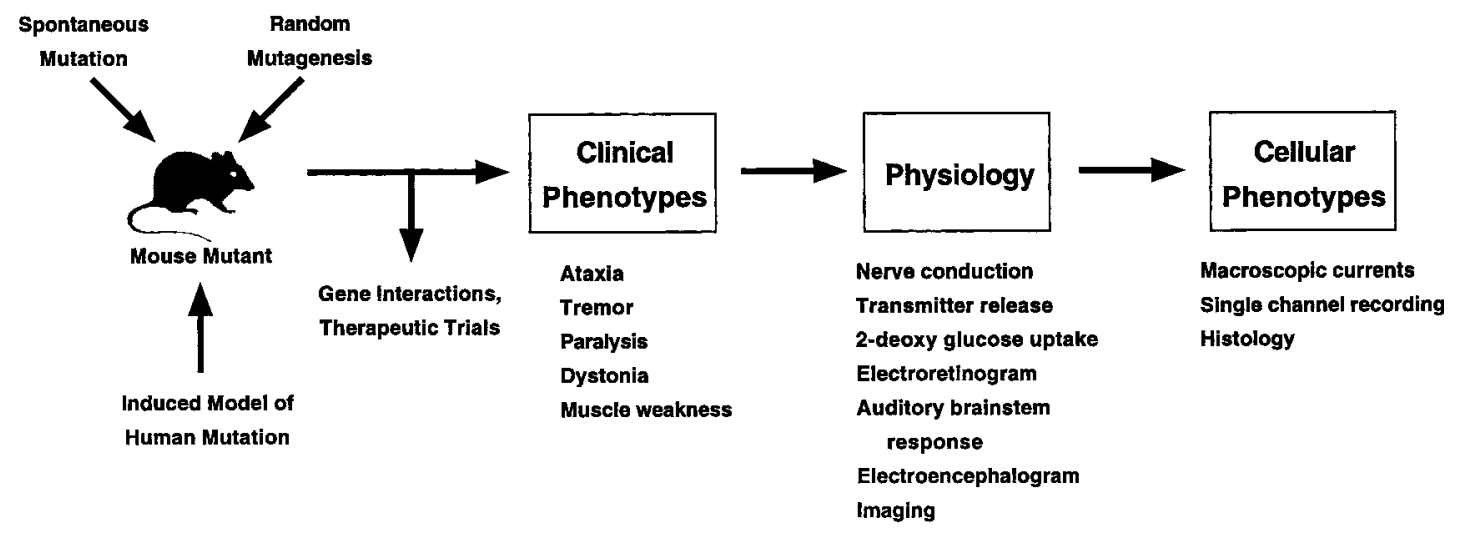

Figure 4. Experimental analysis of neurological dysfunction in mutant mice. The neurological mutations can arise spontaneously, or be introduced to the mouse germ line by chemical mutagenesis in vivo or in ES cells, or by homologus recombination in ES cells. For the case of ion channel mutations, electrophysiological studies on neurons isolated from mutant mice will reflect the in vivo functional alteration of the mutant channel more accurately than assays in Xenopus oocytes or transfected mammalian cells. 
Bournaud, R. \& A. Mallart, 1987. Potassium channel blockers and impulse propagation in murine motor endplate disease. Muscle Nerve 10: 1-5.

Buchner, D.A., M. Trudeau, A.L. George Jr., L.K. Sprunger \& M.H. Meisler, 2003a. High-resolution mapping of the sodium channel modifier Scnml on mouse chromosome 3 and identification of a $1.3-\mathrm{kb}$ recombination hot spot. Genomics 82: 452-459.

Buchner, D.A., M. Trudeau \& M.H. Meisler, 2003b. SCNM1, a putative RNA splicing factor that modifies disease severity in mice. Science 301: 967-969.

Bulman, D.E., 1997. Phenotype variation and newcomers in ion channel disorders. Hum. Mol. Gen. 6: 1679-1685.

Burgess, D.L., D.C. Kohrman, J. Galt, N.W. Plummer, J.M. Jones, B. Spear \& M.H. Meisler, 1995. Mutation of a new sodium channel gene, $S c n 8 a$, in the mouse mutant 'motor endplate disease'. Nat. Genet. 10: 461-465.

Caldwell, J.H., K.L. Schaller, R.S. Lasher, E. Peles \& S.R. Levinson, 2000. Sodium channel $\mathrm{Na}_{\mathrm{v}} \mathrm{l} .6$ is localized at nodes of Ranvier, dendrites, and synapses. Proc. Natl. Acad. Sci. USA 97: 5616-5620.

Chen, K., L.K. Sprunger, M.H. Meisler, H.J. Haller \& D.A. Godfrey, 1999. Reduced spontaneous activity in the dorsal cochlear nucleus of Scn8a mutant mice. Brain Res. 847: 8589.

Dick, D.J., R.J. Boakes \& J.B. Harris, 1985. A cerebellar abnormality in the mouse with motor end-plate disease. Neuropath. Appl. Neurobiol. 11: 141-147.

Dietrich, P.S., J.G. McGivern, S.G. Delgado, B.D. Koch, R.M. Eglen, J.C. Hunter \& L. Sangameswaran, 1998. Functional analysis of a voltage-gated sodium channel and its splice variant from rat dorsal root ganglia. J. Neurochem. 70: $2262-2272$.

Dipple, K.M. \& E.R.B. McCabe, 2000. Phenotypes of patients with "simple" mendelian disorders are complex traits: thresholds, modifiers and systems dynamics. Am. J. Hum. Genet. 67: 1729-1735.

Duchen, L.W. \& E. Stefani, 1971. Electrophysiological studies of neuromuscular transmission in hereditary 'motor endplate disease' of the mouse. J. Physiol. 212: 535-548.

Duchen, L.W., A.G. Searle \& S.J. Strich, 1967. An hereditary motor end-plate disease in the mouse. J. Physiol. 189: 4-6

Escayg, A., B.T. MacDonald, M.H. Meisler, S. Baulac, G. Huberfeld, I. An-Gourfinkel, A. Brice, E. LeGuern, B. Moulard, D. Chaigne, C. Buresi \& A. Malafosse, 2000. Mutations of SCN1A, encoding a neuronal sodium channel, in two families with GEFS + 2. Nat. Genet. 24: 343345.

Füchtbauer, E.M., 1987. Nerve transplantation shows that motor end-plate disease is not a primary Schwann cell defect. Exp. Neurol. 97: 135-142.

Garcia, K.D., L.K. Sprunger, M.H. Meisler \& K.G. Beam, 1998. The sodium channel Scn8a is the major contributor to the postnatal developmental increase of sodium current density in spinal motoneurons. J. Neurosci. 18: 5234-5239.

Harris, J.B., R.J. Boakes \& J.A. Court, 1992. Physiological and biochemical studies on the cerebellar cortex of the murine mutants "jolting" and "motor end-plate disease". J. Neurol. Sci. 110: 186-194.

Kearney, J.A., D.A. Buchner, G. de Haan, M. Adamska, S.I. Levin, A.R. Furay, R.L. Albin, J.M. Jones, M. Montal,
M.J. Stevens, L.K. Sprunger \& M.H. Meisler, 2002. Molecular and pathological effects of a modifier gene on deficiency of the sodium channel Scn8a $\left(\mathrm{Na}_{\mathrm{v}} 1.6\right)$. Hum. Mol. Genet. 11: 2765-2775.

Kohrman, D.C., N.W. Plummer, T. Schuster, J.M. Jones, W. Jang, D.L. Burgess, J. Galt, B.T. Spear \& M.H. Meisler, 1995. Insertional mutation of the motor endplate disease (med) locus on mouse chromosome 15. Genomics 26: 171177.

Kohrman, D.C., J.B. Harris \& M.H. Meisler, 1996a. Mutation detection in the med and med $^{\mathrm{j}}$ alleles of the sodium channel Scn8a: unusual patterns of exon skipping are influenced by a minor class AT-AC intron. J. Biol. Chem. 271: 1757617581.

Kohrman, D.C., M.R. Smith, A.L. Goldin, J.B. Harris \& M.H. Meisler, 1996b. A missense mutation in the sodium channel gene $\mathrm{Scn} 8 \mathrm{a}$ is responsible for cerebellar ataxia in the mouse mutant jolting. J. Neurosci. 16: 5993-5999.

Krzemien, D.M., K.L. Schaller, S.R. Levinson \& J.H. Caldwell, 2000. Immunolocalization of sodium channel isoform NaCh6 in the nervous system. J. Comp. Neurol. 420: 70-83.

Maurice, N., T. Tkatch, M.H. Meisler, L.K. Sprunger \& D.J. Surmeier, 2001. $\mathrm{D}_{1} / \mathrm{D}_{5}$ dopamine receptor activation differentially modulates rapidly inactivating and persistent sodium currents in prefrontal cortex pyramidal neurons. J. Neurosci. 21: 2268-2277.

Meisler, M.H., J. Galt, J. Weber, J.M. Jones, D.L. Burgess \& D.C. Kohrman, 1997. Isolation of mutated genes from transgene insertion sites, pp. 505-520 in Microinjection and Transgenesis of Cultured Cells and Embryos, edited by A. Cid \& Garcia-Carranca, Springer, New York, NY.

Planells-Cases, R., M. Caprini, J. Zhang, E.M. Rockenstein, R.R. Rivera, C. Murre, E. Masliah \& M. Montal, 2000. Neuronal death and perinatal lethality in voltage-gated sodium channel alpha (II)-deficient mice. Biophys. J. 78: 2878-2891.

Plummer, N.W. \& M.H. Meisler, 1999. Evolution and diversity of the mammalian voltage-gated sodium channels. Genomics 57: 323-331.

Plummer, N.W., J. Galt, J.M. Jones, D.L. Burgess, L.K. Sprunger, D.C. Kohrman \& M.H. Meisler, 1998. Exon organization, physical mapping, and polymorphic intragenic markers for the human neuronal sodium channel gene SCN8A. Genomics 54: 287-296.

Raman, I.M., L.K. Sprunger, M.H. Meisler \& B.P. Bean, 1997. Altered subthreshold sodium currents and disrupted firing patterns in Purkinje neurons of Scn8a mutant mice. Neuron 19: 881-891.

Ramesh, T.M., A.W. Ellis \& B.T. Spear, 1995. Individual mouse alpha-fetoprotein enhancer elements exhibit different patterns of tissue-specific and hepatic position-dependent activities. Mol. Cell Biol. 15: 4947-4955.

Rieger, F., M. Pincon-Raymond, A. Lombet, G. Ponzio, M. Lazdunski \& R.L. Sidman, 1984. Paranodal dysmyelination and increase in tetrodotoxin binding sites in the sciatic nerve of the motor end-plate disease (med/med) mouse during postnatal development. Dev. Biol. 101: 401-409.

Schaller, K.L. \& J.H. Caldwell, 2000. Developmental and regional expression of sodium channel isoform $\mathrm{NaCh} 6$ in the rat central nervous system. J. Comp. Neurol. 420: 84 97. 
Schaller, K.L., D.M. Krzemien, P.J. Yarowsky, B.K. Krueger \& J.H. Caldwell, 1995. A novel, abundant sodium channel expressed in neurons and glia. J. Neurosci. 15: 32313242.

Sidman, R.L., J.S. Cowen \& E.M. Eicher, 1979. Inherited muscle and nerve diseases in mice: a tabulation and commentary. Ann. NY Acad. Sci. 317: 497-505.

Smith, M.R. \& A.L. Goldin, 1999. A mutation that causes ataxia shifts the voltage-dependence of the Scn8a sodium channel. Neuroreport 10: 3027-3031.

Spear, B.T., 1994. Mouse alpha-fetoprotein gene $5^{\prime}$ regulatory elements are required for postnatal regulation by raf and Rif. Mol. Cell. Biol. 14: 6497-6505.

Sprunger, L.K., A. Escayg, S. Tallaksen-Greene, R.L. Albin \& M.H. Meisler, 1999. Dystonia associated with mutation of the neuronal sodium channel $S c n 8 a$ and identification of the modifier locus Scnm1 on mouse chromosome 3. Hum. Mole. Genet. 8: 471-479.

Trudeau, M.M., J.D. Dalton, J.W. Day, L.P.W. Ranum \& M.H. Meisler, 2004. Heterozygosity for a truncation allele of sodium channel SCN8A in a family with ataxia and cognitive impairment. Am. J. Hum. Genet. electronic supplement at www.ashg.org

Tzoumaka, E., A.C.S.L. Tischler, R.M. Eglen, J.C. Hunter \& S.D. Novakovic, 2000. Differential distribution of the tetrodotoxin-sensitive $\mathrm{rPN} 4 / \mathrm{NaCh} 6 / \mathrm{Scn} 8 \mathrm{a}$ sodium chanel in the nervous system. J. Neurosci. Res. 60: 37-44.

Weiss, L.A., A. Escayg, J.A. Kearney, M. Trudeau, B.T. MacDonald, M. Mori, J. Reichert, J.D. Buxbaum \& M.H. Meisler, 2003. Sodium channels SCN1A, SCN2A and $S C N 3 A$ in familial autism. Mol. Psychol. 8: 186-194.

Note added in proof: Three new ENU-induced alleles of Scn8a that cause recessive disease have recently been described (Buchner et al., Mammalian Genome 15: 344-351, 2004). A floxed allele for conditional inactivation of Scn8a has been generated (Levin and Meisler, Genesis, 2004, in press). 